\title{
EXPLAINING COMPACT GROUPS AS CHANCE ALIGNMENTS
}

\author{
Gary A. Mamon \\ DAEC, Observatoire de Meudon \\ Meudon, France
}

The physical nature of the apparently densest groups of galaxies, known as compact groups is a topic of some recent controversy, despite the detailed observations (Hickson, in these proceedings, and references therein) of a well-defined catalog of 100 isolated compact groups compiled by Hickson (1982). Whereas many authors have espoused the view that compact groups are bound systems, typically as dense as they appear in projection on the sky (e.g., Williams \& Rood 1987; Sulentic 1987; Hickson \& Rood 1988), others see them as the result of chance configurations within larger systems, either in 1D (chance alignments: Mamon 1986; Walke \& Mamon 1989), or in 3D (transient cores: Rose 1979). As outlined in the companion review to this contribution (Mamon, in these proceedings), the implication of Hickson's compact groups (hereafter, HCGs) being dense bound systems is that they would then constitute the densest isolated systems of galaxies in the Universe and the privileged site for galaxy interactions.

In a previous paper (Mamon 1986), I reviewed the arguments given for the different theories of compact groups. Since then, a dozen papers have been published on the subject, including a thorough and perceptive review by White (1990), thus more than doubling the amount written on the subject. In this contribution, I first enumerate the arguments that I brought up in 1986 substantiating the chance alignment hypothesis, then review the current status of the numerous recent arguments arguing against chance alignments and/or for the bound dense group hypothesis (both for the majority of HCGs but not all of them), and finally reconsider each one of these "anti-chance alignment" arguments and show that, rather than being discredited, the chance alignment hypothesis remains a fully consistent explanation for the nature of compact groups.

\section{Old Arguments for HCGs as Chance Alignments}

I start by listing the arguments I gave in Mamon (1986) to suggest that most HCGs are caused by chance alignments of galaxies along the line of sight within looser systems.

\section{Frequency of chance alignments}

From my dynamical simulations of loose groups (hereafter, LGs), I estimated the frequency at which a projected configuration showed a subgroup that was compact using Hickson's (1982) criteria and found values ranging from $3 \%$ to $33 \%$, depending on the number of galaxies in the LGs, and on where the dark matter was placed in them (Mamon 1987). I had also estimated the frequency at which compact configurations would have to have appeared in order to explain half of the HCGs as chance alignments, and found it to be $2.5 \%$ (Mamon 1986). The majority of these compact configurations were $1 D$ chance alignments rather than 
3D transient cores (Mamon 1987). Chance alignments were thus numerous enough to explain HCGs and perhaps even too frequent!

\section{Demographics}

As mentioned in my companion review, dense groups formed in less than $5 \%$ of the LGs simulated for a Hubble time, while I argued that a frequency of 6 to $45 \%$ of dense group occurrence within LGs was necessary (Mamon 1986). Thus, dense groups form too rarely to explain most HCGs.

Moreover, if HCGs are bound dense groups then they must rapidly coalesce into giant ellipticals or $\mathrm{cDs}$, and I estimated that 5 to $40 \%$ of all such galaxies would then be the products of multiple mergers in dense groups (Mamon 1986, see also Barnes 1989). Williams \& Rood (1987) argue that there ought to be more coalesced dense groups than observed giant ellipticals, and are thus led to worry about Newtonian theory as a whole.

\section{Dynamical evolution}

The large number of simulations performed by Mamon (1987) provided a useful statistical ensemble on which to study dynamical evolution. I found that dense groups rapidly show strong signs of luminosity segregation and evolution at the bright-end of the galaxy luminosity function. These are not seen in HCGs (Mamon 1986) thus questioning the bound dense group hypothesis.

\section{HCG galaxy morphological types}

The morphology-density relation linking the mix of galaxy morphologies to the local galaxy density, found for groups and clusters (e.g., Postman \& Geller 1984) is not obeyed for HCGs (assuming that they are as dense in 3D as they appear), which follow their own relation, offset from the "universal" one by a factor of 200 in local galaxy number density. This is precisely what one predicts if HCGs are caused by chance alignments in looser groups, thus having the same mix of morphologies as their parent groups but appearing very much denser.

\section{5. $H C G$ mass-to-light ratios}

Buckley \& Mamon (1987, unpublished) and Hickson (quoted in White 1990) find the median HCG virial or projected mass-to-light ratio to be around $40 h$, which is much smaller than the typical LG values (Tully 1987 and Ramella et al. 1989 find the median LG M/L to be $125 h$ and $180 h$, respectively). Now if HCGs are chance alignments within LGs they should have the same velocity dispersion as their parent groups, and one should then expect that the ratio of $\mathrm{HCG}$ to $\mathrm{LG}$ median $\mathrm{M} / \mathrm{L}$ is roughly equal to the corresponding ratio of sizes divided by that of total luminosities. Now, the ratio of total luminosities is $\simeq 2$. If Tully's groups are typical of HCG parents, then the ratio of sizes is 17 , but if HCGs arise from typically denser LGs (Walke \& Mamon 1989) then from the morphology-density relation offset, the ratio is 6 . For the latter case one then obtains precisely the median HCG M/L expected. Alternatively, a low median M/L may be expected if HCGs are bound dense systems, extending much less than the dark matter, for example if they are part of looser groups.

\section{Arguments for HCGs as Dense Bound Groups}

There are many arguments pointing to the idea that what you see is what you get, i.e., that HCGs are as dense in 3D as they appear in 2D. I list these below.

\section{Frequency of chance alignments}


Hickson \& Rood (1988) have performed static Monte-Carlo simulations of galaxy positions in a group, by placing points at random in a circle or a sphere, and computing the probability of an isolated compact subgroup occuring by chance (in projection when they start in the sphere). For typical LG parameters they find a chance alignment probability of $10^{-5}$ or $5 \times 10^{-4}$, depending on whether the typical parent LG has a binary within it or not. Their results seem inconsistent with the probabilities of 2 to $25 \%$ that I had found in my dynamical simulations (Mamon 1987), and moreover discredit the whole chance alignment phenomenon as much too rare to explain a significant fraction of HCGs.

\section{HCG environments}

By definition, HCGs are isolated: Denoting the angular radius of the smallest circumscribed circle containing the centers of the galaxies in an HCG by $\theta_{\mathrm{HCG}}$ and the magnitude of the brightest galaxy by $m_{1}$, there must be no galaxies brighter than $m_{1}+3$ in a concentric annulus of angular radii $\theta_{\mathrm{HCG}}$ and $3 \theta_{\mathrm{HCG}}$. Various authors have attempted to measure the isolation of an HCG in a wider environment. If an HCG is isolated, then it is unlikely the result of chance alignments within looser systems. For Williams \& Rood (1987), an HCG is isolated if there are fewer than four outside galaxies in the magnitude range $\left(m_{1}, m_{f}\right)$ of the HCG members, within a distance of $3 \theta_{\mathrm{HCG}}$ from any of the HCG galaxies, and thus find 89 isolated HCGs. Sulentic (1987) considers the surface number density of galaxies outside of the HCG out to $\theta=0.5^{\circ}$ and $1^{\circ}$, and brighter than $m_{f}+1$. He concludes that HCGs have a surface number density 100 times greater than their immediate environment, and calls isolated the 38 HCGs whose immediate environment is less dense than the average field. Rood $\&$ Williams (1989) count the galaxies out to $10 \theta_{\mathrm{HCG}}$ with mags in the range $m_{1}$ to $m_{f}$, and compare to the estimated field surface number density based on a model of galaxy counts (why not use the observed field counts instead?). They find 67 HCGs whose environments have a density that is less than $2 \sigma$ above the expected field surface number density.

\section{HCG galaxy morphological types}

Sulentic (1987) found that the mix of morphological types of the galaxies in HCGs was roughly the same as in their environments (out to both $0.5^{\circ}$ and $1^{\circ}$ ). However, Rood \& Williams (1989) find that the environments of HCGs (out to $10 \theta_{\mathrm{HCG}}$ ) are more spiral rich than the HCGs (whose morphological types were determined by Williams \& Rood 1987), hence the HCGs are more evolved.

For Hickson \& Rood (1988), the offset of the HCG morphology-density relation is caused by a stronger correlation of galaxy morphologies with the velocity dispersion of the group or cluster to which they belong. Their reasoning is based upon trends found in HCGs by Hickson, Kindl, \& Huchra (1988), but with only global mean values for LGs and clusters. Such a morphology-velocity dispersion relation, if true, would be a fundamental clue to galaxy formation and evolution, but this has to be studied in more detail for LGs and clusters.

Finally there is a significant tendancy for the morphological types of individual galaxies within an HCG to agree among one another (Hickson, Kindl, \& Huchra 1988; see also Sulentic 1987), which seems to indicate that HCGs are specific isolated systems.

\section{4. $H C G$ elongations}

Sargent \& Turner (1972, unpublished) and Rose (1977) studied the distribution of the elongations of compact groups, and both argued that the shapes of compact groups were consistent with random configurations viewed in projection, i.e., chance alignments perpendicular to the line of sight. This issue was addressed again by Hickson et al. (1984), who 
showed that the distribution of HCG elongations was inconsistent with random sampling from a sphere or a disk, in that there were too many observed highly elongated groups.

\section{Demographics}

Assuming that bound dense groups are created and destroyed at the same rate, Barnes (1989) has argued that the ratio of the number of dense groups to the number of LGs in the same volume of the Universe should be equal to the ratio of their lifetimes, which is close to the ratio of their crossing times, i.e., about 1\%. Hence, he explains HCGs as bound dense groups forming within LGs by two-body interactions and being destroyed by mergers.

\section{Galaxy interaction in $H C G s$}

This is perhaps the most studied point, and I thus save the best for the end. Numerous studies have observed signs of galaxy interaction in HCGs, thus seemingly arguing for HCGs as bound dense groups. Menon \& Hickson $(1985,1990)$ have shown that continuum radio sources in HCGs are always attached to individual galaxies, but whereas a spiral of any luminosity rank in its group can be a radio-source, an elliptical or lenticular must almost always be a first-ranked group member. Williams \& Rood (1987) have argued that HCGs are deficient in HI. Hickson et al. (1989) found significant IR emission in HCG galaxies. Rubin, Hunter, \& Ford (1990) found peculiar rotation curves in 14 of the 21 spiral galaxies they observed. Zepf \& Whitmore (1990) argued that.many HCG ellipticals (usually faint ones) are bluer than expected.

\section{Discussion}

I now reconsider each one of the arguments given in the preceding section.

\section{Frequency of chance alignments}

The frequency (instead of probability since one can have more than one compact subgroup within a large enough group) of chance alignments was reevaluated by Walke \& Mamon (1989), who solve analytically the problem of forming isolated compact subgroups from a parent homogeneous distribution in a circle (the same problem as numerically simulated by Hickson \& Rood). They find that the frequency of chance alignments is very sensitive to the size of the parent $\mathrm{LG}$, typically varying as $R_{\mathrm{LG}}^{-4.5}$, and also increases rapidly with the membership of the parent LG. Their computed frequencies are consistent with both Mamon's (1987) dynamically simulated results as well as Hickson \& Rood's very low values! The difference is caused by the relatively small initial sizes of my dynamically simulated LGs, and the fact that I searched for configurations that met the HCG selection criteria, while Hickson \& Rood attempted to match the median HCG properties (the HCG sample turns out to be very incomplete at the faintest two mags $\operatorname{arcsec}^{-2}$ in surface magnitude). Moreover, Walke \& Mamon argue that while the median loose group has a negligible frequency of chance alignments, the mean group does not, so that summing over an LG catalog (Tully 1987), they find a mean frequency of chance alignments of $7 \%$, which remains as high as $1 \%$ if they exclude the Virgo cluster from the LG catalog. And such a one-percent frequency is what is required to account for roughly half of HCGs being caused by such chance alignments (within a factor of four). Finally, they suggest that clusters ought to contain compact groups embedded within them, and Mamon (1989) discovered such a compact group in the Virgo cluster by performing an automated search of isolated subgroups strictly satisfying the HCG selection criteria. 


\section{2. $H C G$ environments}

Do the closest and brightest HCGs belong to known loose groups or clusters? I've searched for membership in larger systems of all HCGs with at least two member galaxies with similar redshifts $\left(\Delta v<1000 \mathrm{~km} \mathrm{~s}^{-1}\right)$ included in a galaxy redshift survey (the remaining HCG galaxies being out of the boundaries of the galaxy survey, or too faint to be included in it). There were five such HCGs. All of them were found to belong to loose groups, as shown in Table 1.

\section{Table 1: Nearby HCGs}

\begin{tabular}{|c|c|c|c|c|c|}
\hline HCG \# & $N$ & Embedding group & WR87 & $\begin{array}{l}\text { Isolation } \\
\text { S87 }\end{array}$ & RW89 \\
\hline $\begin{array}{r}44 \\
58 \\
61 \\
68 \\
90 \\
101\end{array}$ & $\begin{array}{l}4 \\
5 \\
3 \\
5 \\
4 \\
5\end{array}$ & $\begin{array}{c}\text { GH } 58(10), \text { NBG } 21-6(12) \\
\text { GH } 89(7), \text { MKW } 10(21) \\
\text { GH } 101(11), \text { RGH } 33(6) \\
\text { GH } 123(17), \text { NBG } 42-1(15) \\
\text { NBG } 63-1(6) \\
\text { GH } 106(248), \text { NBG } 11-1(130)\end{array}$ & $\begin{array}{l}\text { Isolated } \\
\text { Isolated } \\
\text { Isolated } \\
\text { Isolated } \\
\text { Isolated } \\
\text { Isolated }\end{array}$ & $\begin{array}{l}\text { Isolated } \\
\text { Non-isolated } \\
\text { Isolated } \\
\text { Isolated } \\
\text { (Isolated) } \\
\text { Isolated }\end{array}$ & $\begin{array}{l}\text { Non-isolated } \\
\text { Non-isolated } \\
\text { Isolated } \\
\text { Non-isolated } \\
\text { Non-isolated } \\
\text { Non-isolated }\end{array}$ \\
\hline
\end{tabular}

Notes: $N$ is the number of galaxies with accordant redshifts, WR87, S87, and RW89, are the studies of Williams \& Rood (1987), Sulentic (1987), and Rood \& Williams (1989), and GH, NBG, MKW, and RGH stand for the galaxy systems of Geller \& Huchra (1983), Tully (1987), Morgan, Kayser \& White (1975), and Ramella, Geller, \& Huchra (1989), respectively. The number in parentheses is the number of galaxies in the embedding system. I also list as HCG 101 the compact group that I discovered in the Virgo cluster (Mamon 1989).

In addition to these, Tikhonov (in these proceedings) found six more HCGs within LGs. Sulentic (1987) would classify all of the HCGs listed in Table 1 as isolated, as the parent groups or cluster span far beyond his angular limits. But more surprising is the fact that Williams \& Rood (1987) would have classified 5 out of 6 of these compact groups as isolated. On the other hand Rood \& Williams (1989) would classify only one of the 6 compact groups as isolated, which tends to suggest that their isolation criterion is much better although not perfect. It would not be surprising that once deeper redshift surveys are established, the majority of HCGs would belong to greater 3D structures. Note however that while this saves the chance alignment hypothesis, it does not prove it since, if HCGs are bound dense systems, they would have to form within greater structures (see my review on dense groups in these proceedings).

\section{3. $H C G$ galaxy morphological types}

Tikhonov (these proceedings) finds a significantly smaller fraction of spirals in more distant HCGs, thus suggesting that ellipticals are oversampled. I checked this using the very accurate morphologies based upon CCD frames given in Hickson, Kindl, \& Auman (1989): HCG galaxies are $24 \pm 5 \%$ spiral at $z \geq 0.04$, compared to $56 \pm 4 \%$ for $z<0.04$, a $\simeq 6 \sigma$ result. It thus seems preferable to exclude the 24 HCGs with $z>0.04$ when analysing morphologies.

Once Rood \& Williams divide their HCG sample into the isolated and non-isolated HCGs (see previous section), they find that the morphologies are not significantly different between the 67 isolated HCGs and their sparse environments. A significant difference is present for the 33 remaining HCGs and their neighborhoods, but only for this minority. 
White (1990) has argued that morphological concordance is the result of similar conditions at galaxy formation, and linked to a correlation of galaxy morphological types with some yet unknown physical quantity. I find that the quartets in the CfA LG catalog (Geller \& Huchra 1983) present no such significant morphological concordance: perhaps because the correlation of quantities is less strong in LGs.

\section{4. $H C G$ elongations}

Hickson et al. (1984) also studied the distribution of HCG elongations using simple dynamical models of both groups and subgroups within groups, and found the two distributions statistically consistent. Thus, the issue of dense bound groups versus chance alignments was thus not resolved here, in contrast to what is stated by Hickson \& Rood (1988).

\section{Demographics}

The logic of Barnes is based upon the assumption that on average each loose group sees one (and only one) bound dense group form within it in its lifetime. Now, my simulations of LGs (Mamon 1987) were stopped at a Hubble time, while the LG lifetimes, based upon their crossing times and the merger rates summarized in my review in these proceedings, was about double. Hence, Barnes would expect me to have found bound dense groups within half of my simulated LGs, whereas I had only found roughly $5 \%$, or, in other words, the formation rate of dense groups within LGs is ten times too low to explain HCGs.

\section{Galaxy interaction in $H C G s$}

Now to the fundamental point. If HCGs are caused by chance alignments, then these are not simply well separated individual galaxies lying along the line of sight. A compact quartet, could be such a " $1+1+1+1$ " system, but could also be an alignment of binaries, or a triplet aligned with a single galaxy. The galaxies that are physically associated can moreover be bound to one another or not, for example one could have a transient unbound triplet. The mix of these populations is of fundamental importance in assessing the nature of HCGs, and unfortunately has not been yet looked for in the explicit-physics simulations (where statistical results can be obtained). I thus allowed myself to guess the expected mix for quartets appearing as chance alignments, and these appear in Table 2, where I also used the results of my simulations (Mamon 1987).

\section{Table 2: Approximate distribution of $\mathrm{HCGs}$}

\begin{tabular}{|c|c|c|c|}
\hline \multirow[t]{2}{*}{$\begin{array}{l}\text { Individual Halos } \\
85 \%\end{array}$} & \multicolumn{2}{|c|}{ Chance Alignments } & \multirow[t]{2}{*}{$\begin{array}{c}\text { Common Intergalactic Background } \\
55 \%\end{array}$} \\
\hline & $\begin{array}{c}1+1+1+1 \\
\underline{\mathbf{2}}+1+1 \\
\underline{\mathbf{2}}+\underline{\mathbf{2}} \\
\underline{\mathbf{3}}+\underline{1} \\
\underline{\mathbf{3}}+1\end{array}$ & $\left.\begin{array}{l}35 \% \\
35 \% \\
10 \% \\
10 \% \\
10 \%\end{array}\right)$ & \\
\hline $13 \%$ & \multicolumn{2}{|c|}{ Transient Cores } & \multirow[t]{2}{*}{$40 \%$} \\
\hline & & $100 \%$ & \\
\hline $2 \%$ & \multirow{2}{*}{\multicolumn{2}{|c|}{$\begin{array}{c}\text { Bound Dense Groups } \\
4\end{array} \quad 100 \%$}} & $5 \%$ \\
\hline & & & \\
\hline
\end{tabular}

NOTES: The underlined numbers in bold correspond to bound systems of galaxies. 
From the numbers in Table 2, I expect $32 \%$ or $24 \%$ of strongly interacting galaxies, if the dark matter resides in individual halos or a common intergalactic background, respectively. The weak interactors account for an additional $19 \%$ or $44 \%$ of the galaxies for the two dark matter situations, respectively. So a chance alignment model of HCGs turns out to be fully consistent with galaxy interactions. As a cautionary note, in the one dense group simulation of Barnes (1989), mergers occur mainly at the beginning and the end of the simulation, and little interaction is seen during most of the life of the group.

Now the predicted existence of galaxy interactions in chance aligned HCGs should affect somewhat the mix of morphologies in these systems relative to their environments, and might thus explain the differences discovered by Rood \& Williams (1989).

\section{Future Prospects}

In summary, while a minority of HCGs display strong signs of interaction, these are consistent with chance alignments, which moreover are well justified from statistical arguments. While the controversy surrounding the nature of compact groups will certainly not end soon, there are quite a few tests that ought to be tried out, which I list below.

The explicit-physics simulations that I've carried out (Mamon 1987), although inaccurate in reproducing the details of the galaxy interactions, are probably the best way to assess the amount of binaries and triplets in chance aligned quartets and quintets. The distribution of group elongations can also be properly assessed in this way. One can also test the distribution of mass-to-light ratios of the compact configurations occurring in projected simulated loose groups, and compare with the HCG sample.

More detailed simulations like the restricted 3-body simulations being undertaken by Borne \& Levison (in preparation) are then needed to obtain statistical estimates of the strength and the duration of galaxy interactions in dense groups, and in loose groups as well. In the long run, one will of course strive for a statistical set of self-consistent dense group simulations, perhaps with 1000 particles per galaxy.

Observationally, the day will come when POSS plates will be automatically scanned and standard group as well as HCG algorithms can be applied to these. We will thus find out if compact groups are always situated in looser groups or in clusters, and the importance of the latter environment. We would also have better statistics to assess the difference in morphologies.

\section{References}

Barnes, J. 1989, Nature, 338, 123.

Geller, M.J., and Huchra, J.P. 1983, Ap. J. Suppl., 52, 61.

Hickson, P. 1982, Ap. J., 255, 382.

Hickson, P., Kindl, E., and Auman, J.R. 1989, Ap. J. Suppl., 70, 687.

Hickson, P., Kindl, E., and Huchra, J.P. 1988, Ap. J., 331, 64.

Hickson, P., Menon, T.K., Palumbo, G.G.C., and Persic, M. 1989, Ap. J., 341, 679.

Hickson, P., Ninkov, Z., Huchra, J.P., and Mamon, G.A. 1984, in Clusters and Groups of Galaxies, ed. F. Mardirossian, G. Giuricin, and M. Mezzetti (Dordrecht: Reidel), p. 367.

Hickson, P. and Rood, H.J. 1988, Ap. J. (Letters), 331, L69.

Mamon, G.A. 1986, Ap. J., 307, 426.

Mamon, G.A. 1987, Ap. J., 321, 622.

Mamon, G.A. 1989, Astr. Ap., 219, 98. 
Menon, T.K., and Hickson, P. 1985, Ap. J., 296, 60.

Menon, T.K., and Hickson, P. 1990, in preparation.

Morgan, W.W., Kayser, S., and White, R.A. 1975, Ap. J., 199, 545.

Postman, M., and Geller, M.J. 1984, Ap. J., 281, 95.

Ramella, M., Geller, M.J., and Huchra, J.P. 1989, Ap. J., 344, 57.

Rood, H.J., and Williams, B.A. 1989, Ap. J., 339, 772.

Rose, J.A. 1977, Ap. J., 211, 311.

Rose, J.A. 1979, Ap. J., 231, 10.

Rubin, V.C., Hunter, D., and Ford, W.K. 1990, in preparation.

Sulentic, J.W. 1987, Ap. J., 322, 605.

Tully, R.B. 1987, Ap. J., 321, 280.

Walke, D.G., and Mamon, G.A. 1989, Astr. Ap., 295, 291.

White, S.D.M. 1990, in Dynamics and Interactions of Galaxies, ed. R. Wielen, in press.

Williams, B.A., and Rood, H.J. 1987, Ap. J. Suppl., 63, 265.

Zepf, S.E., and Whitmore, B.C., 1990, in preparation.

\section{DISCUSSION}

Sulentic: Three comments: 1) The failure of simulations to produce dense groups is not a basis for rejecting that they are physical systems. 2) I am surprised by the lack of infrared emission from the dense groups - it supports your view. I believe that Hickson et al. overestimate the FIR luminosities of group members (due to resolution of IRAS). 3) At least some dense groups do have luminous haloes. At least 3 are discordant groups-your model would increase the significance of these associations because these 3 groups would populate the reduced sample of physical groups.

Mamon: If F. Hammer were here, I guess he would respond to your last comment by arguing that the discordant member, if it is a background object, would be gravitationally lensed by the remaining group. This would amplify it enough to make it almost as bright as the other group members. Perhaps gravitational lensing of the background object could also be responsible for the diffuse light that you find in some of Hickson's compact groups.

Whitmore: 1 . Vera Rubin has also looked at about a dozen ellipticals in compact groups, and finds that most of them have emission in $\mathrm{H} \alpha$, and one case is a counterrotating system. 2. I recently reexamined the morphology-density relation using Dressler's data for 55 clusters. I found that the fundamental correlation is probably with the distance from the center of the cluster rather than local density (i.e.. - high fractions of ellipticals are only found near the centers of clusters). This would explain why the morphology-density relation is offset for compact groups (i.e., not all ellipticals as would be predicted by high local density) without implying they are not physically related. The small number of galaxies in compact groups cannot produce the deep potential well found in clusters with hundreds of galaxies. 
Hamon: I believe that loose groups cannot produce deep potential wells like those found in clusters. However, there is a morphology-density relation on loose groups (e.g., Postman \& Geller) which follows that of clusters. So one must explain the offset of Hickson's compact groups relative to loose groups in the morphology-density relation.

Hickson: I think that you and $I$ both agree that the probability of a sufficiently compact chance alignment of galaxies occuring in a typical loose group is extremely small. When you include the virgo cluster you find a mean probability which is about an order of magnitude higher than when Virgo is excluded. This means that if compact groups are mostly chance alignments, they should almost all be in clusters as least as rich as virgo. Yet very few of the compact groups in my catalog are anywhere near such clusters.

Mamon: One should therefore perform automated searches inside clusters (with $N \geqslant 50$ accordant redshifts) such as the one I performed on Virgo, and one should perhaps expect to find a large number of new HCG's in such clusters. 